\title{
Clinical assessment is sufficient to allow outcome evaluation following surgical management of Achilles tendon ruptures
}

\author{
Atanas Todorov \\ Frederic Schaub \\ Fabian Blanke \\ Patricia Heisterbach \\ Franciska Sachser \\ Andreas Gösele \\ Martin Majewski
}

Atanas Todorov and Frederic Schaub are equal contributing first authors.

Department of Orthopedic Surgery, University of Basel, Switzerland

Corresponding author:

Atanas Todorov

Department of Orthopedic Surgery, University of Basel

Spitalstrasse 21

4031 Basel, Switzerland

E-mail: atanas.todorovjr@usb.ch

\section{Summary}

Study design: cross-sectional study in otherwise healthy athletic adults with a unilateral Achilles tendon rupture.

Objectives: define the relationships of active range of motion, calf circumference or number of heel raises to a full set of isokinetic parameters.

Background: Achilles tendon ruptures commonly occur during sports and create a considerable amount of morbidity. The benefits of different treatments are difficult to determine. Complex and expensive isokinetic testing is often required. If a simple force measurement could replace this testing, large clinical trials would be more easily feasible.

Methods: 74 patients with acute Achilles tendon rupture and surgical treatment were evaluated retrospectively. Active range of motion (ROM), ratio of ROM, number of heel raises, ratio of heel raises, calf circumference and isokintetic measurements were recorded. Regression using a Bayesian elastic net showed the most important correlations.

Results: Active range of motion showed a significant correlation to peak torque angle at flexion and extension as well as increased sports activity. There was a negative correlation to percuta- neous therapy. Active Heel raise showed a positive correlation to peak torque at dorsal extension and increased sports activity as well as a negative correlation to high postoperative pain, where as calf circumference was positive correlated to peak torque at dorsal extension and body height as well as negative correlated to female gender. Conclusion: device independent measures, like range of Motion, and amount of Heel raise, are an excellent tool providing similar information compared to isokinetic testing and could be used to evaluate clinical outcome after Achilles tendon rupture.

KEY WORDS: Achilles tendon rupture, outcome evaluation, surgical treatment.

\section{Introduction}

Achilles tendon ruptures most commonly occur during sports and are responsible for a considerable amount of morbidity among the young to middle aged involved in leisure sports with a reported annual incidence of 18 per $100,000^{1,2}$. Treatment options range from conservative therapy to open surgery ${ }^{3-5}$. It is difficult to determine the true benefit of a treatment. For clinical practice some objective and subjective measurements have been developed $^{6,7}$. These include dorsal flexion, plantar flexion, calf size, heel raise, patient-reported function and pain ${ }^{8}$. However, the most commonly used scores based on these parameters unfortunately lack a rigorous validation $^{9}$. More complex isokinetic force measurements are unquestionably valid and provide a multitude of variables ${ }^{8,10}$. However, they are expensive, effortful, difficult to interpret and unpractical in routine patient follow-up. Comparing simple and feasible measurements on one hand and a vast array of biophysical variables on the other is not trivial. Ideally the method used for comparison should indicate the most important correlations and highlight their magnitude. One has to keep in mind that the number of patients is often much less than the number of parameters measured. Linear regression with a Bayesian elastic net can extract the most relevant variables. In this way, a clinician could derive valid conclusions about a therapy with the simplest tools: his hands, a goniometer and a wall. Therefore the present study sought to define the relationship between simple force related measurements - active dorsal flexion and plantar flexion, calf circumference, heel raise - and a full set of isokinetic measurements to prove their clinical importance, correlation and validity. 


\section{Materials and methods}

This study was conducted in accordance with international ethical guidelines as described in Padulo et al. ${ }^{11}$. It was approved by the institutional review board of the University Hospital Basel (EKBB). All patients gave informed consent in order to participate.

Patients who were treated for acute Achilles tendon rupture at the hospitals Basel and Liestal during the period 1996-2006 were retrospectively evaluated. The inclusion criterion was a unilateral rupture of the Achilles tendon treated by percutaneous or open surgery. Exclusion criteria were any other pathology of the ipsilateral or contralateral foot, secondary injuries, fractures, surgeries on the lower extremities, degenerative changes or osteoarthritis of the lower extremities, rheumatoid arthritis and neurological or cardiopulmonary problems preventing biomechanical testing. Out of 162 identified patients 74 patients were included. The age ranged from 29-46 years ( $\varnothing 37$ years). 16 women and 58 men participated. Measurements were performed $55( \pm 19,6)$ months after operation.

\section{Surgical techniques}

Percutaneous repair was done in a modified technique according to Webb and Bannister ${ }^{12}$. Patients lay prone on the operating table and skin incisions were marked. The leg was prepared and draped in the usual manner. The first incision was made at the site of the rupture, the second $5 \mathrm{~cm}$ proximal to this and the third $5 \mathrm{~cm}$ distal. A small curved hemostat was used to define the track into the tendon itself. A single loop of a No. 1 nylon suture on a curved $90 \mathrm{~mm}$ cutting needle is passed through the middle incision then through the tendon substance, exiting at the proximal incision. The needle is reintroduced through the proximal incision, ensuring that a separate entry point in the tendon is used, and taken out through the middle incision. It is then introduced into the middle incision and the distal stump of the tendon is captured. An artery clip is placed on the free ends of the suture before a second one is passed. A hemostat ensures that the sutures are not catching skin and subcutaneous tissues. The ankle is placed in plantar flexion while the sutures are tied.

For open surgery a longitudinal incision was made $1 \mathrm{~cm}$ medial to the Achilles tendon, preserving the lesser saphenous vein and the sural nerve. Dissecting between the Achilles tendon and the paratenon created a full thickness flap. The tendon rupture was identified and the edges minimally debrided. Repair of the tendon was achieved using either the Bunnell or modified Kessler method with number five non-absorbable suture and a running epitendinous absorbable 2.0 suture. Careful repair of the paratenon was performed in all cases. A plaster cast in approximately $20^{\circ}$ plantar flexion was applied.

\section{Examination}

Every patient was tested for active plantar flexion and dorsal extension of both ankle joints. The measurements were performed with the knee flexed at $0^{\circ}$ and $90^{\circ}$. Ratio of dorsal extension and plantar flexion of affected and unaffected leg were calculated. Calf circumference of both legs was measured $10 \mathrm{~cm}$ below the knee and differences of calf circumference between affected and unaffected leg were defined. Heel raises were performed while standing on one foot and keeping the balance by lightly touching a rail with the fingertips. The number of heel raises above $3 \mathrm{~cm}$ was recorded. Three measurements were performed, one before warm-up, one after warm-up for 10 minutes on a treadmill and one after isokinetic measurement. Average and total number of heel raises was used for analysis. Ratios of affected to unaffected leg were calculated. Isokinetic measurements of both legs were performed in a seated position with speeds of $45 \% \mathrm{sec}, 60 \% \mathrm{sec}$ and $120 \% \mathrm{sec}$. Range of movement, peak torque, peak torque angle for concentric dorsal flexion and plantar flexion of the foot were recorded. No verbal encouragement was given during the measurements. Four measurements were performed and the average was used in further analysis. Active dorsal extension, plantar flexion, average heel raises, total heel raises and the ratios were used as dependent variables. Independent variables included the isokinetic parameters, kind of surgery (open or percutaneous), sex, age, height, weight, body mass index (BMI), duration of hospital stay, duration of postoperative sick leave, time to follow up, current pain on a visual analogue scale (VAS), take off leg, dominant leg, sports before and after treatment and changes in sport level after treatment (categorized as more, same or less).

\section{Statistical analysis}

Regression was performed using a Bayesian implementation of the elastic net, a form of a Least Absolute Shrinkage and Selection Operator (LASSO, Tibshirani 1996) ${ }^{13}$, as proposed in Kyung et al. $(2010)^{14}$. Linear regression was used for continuous data, and both poisson and binomial regressions with a random link function were used for heel raise counts. For the intercept a flat prior was used, whereas prior information for the coefficients was taken as the best general linear model estimate and the prior for total variation was uninformative. Simulations were performed using the Open BUGS version 3.2.2 software. Coefficients were considered significant if both the 2.5 and 97.5-percentile of the credible interval had the same sign. The results are given as the coefficient in round brackets followed by the 2.5$97.5 \%$-credible interval in square brackets.

\section{Results}

\section{Range of motion while sitting}

Dorsal extension of the affected foot was negatively correlated with percutaneous surgical treatment $(-1.8$ $[-2.9,-0.7])$, and positively with peak torque angle at flexion of the affected foot with $45^{\circ} / \sec (1.2[0.2,2])$. 


\section{A. Todorov et al.}

Plantar flexion of the affected foot was positively correlated with the peak torque angle at flexion of the affected foot with $45 \%$ sec $(0.8[0.02,1.6])$.

The ratio of dorsal extension was negatively correlated with percutaneous therapy $(-0.05[-0.09,-0.004])$. The ratio of plantar flexion was positively correlated with the peak torque at flexion of the affected foot with $120 \%$ sec $(0.03[0.0006,0.06])$.

\section{Range of motion while standing}

Dorsal extension of the affected foot was not correlated significantly with any variable. Plantar flexion of the affected foot was positively correlated with the peak torque angle at flexion of the affected foot with $45^{\circ} / \mathrm{sec}(1.1[0.1,2])$.

The ratio of dorsal extension was positively correlated with a higher sport level $(0.05[0.007,0.1])$. The ratio of plantar flexion was positively correlated with the peak torque at dorsal flexion of the affected foot with $120 \% \mathrm{sec}(0.02[0.06,0.1])$ and with $60 \% \mathrm{sec}(0.05$ $[0.005,0.09])$. Correlation with percutaneous therapy was negative $(-0.05[-0.09,-0.005])$.

\section{Calf circumference}

The calf circumference of both legs was positively correlated with the peak torque at dorsal extension of

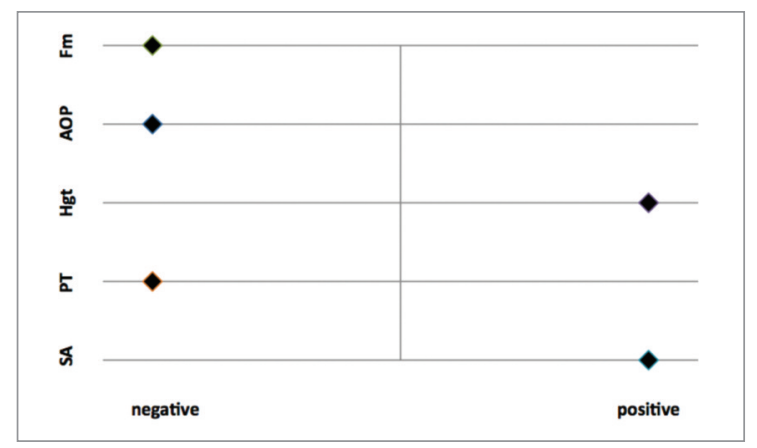

Figure 1. Correlations of device independent measures. Fm: female, AOP: amount of pain, Hgt: body height, PT: percutaneous therapy, SA: sports activity. the affected foot with $120 \% \mathrm{sec}$ and $60 \% \mathrm{sec}$ and with height of the patients. There was a negative correlation if the patient was female. The ratio showed no significant correlations.

\section{Heel Raises}

Average heel raises of the affected foot were negatively correlated with the amount of pain indicated by the patient $(-0.03[-0.05,-0.001])$. Total heel raises were not correlated significantly with any variable. The ratio of average heel raises was not correlated significantly with any variable. The ratio of total heel raises was positively correlated with the peak torque at dorsal extension of the affected foot with $120 \% \mathrm{sec}(0.06[0.03,0.09])$ and with a higher sport level (0.04 [0.0006, 0.09]) (Fig. 1, Tab. 1).

\section{Discussion}

In the present study, we found that the active range of motion both while sitting and standing showed a significant correlation to peak torque angle and peak torque of plantar flexion. No other measurement showed a direct correlation with plantar flexion.

Appropriate calf muscle strength and calf muscle endurance are crucial for a normal gait cycle, ability to walk on tiptoe and the performance of heel-raises. Even during daily activities the calf muscles should be able to generate high peak torques at the ankle joint ${ }^{8}$. Therefore the most crucial parameters for the Achilles tendon are the peak torque and peak torque angle of plantar flexion. Because of this, an isokinetic dynamometer is used in the outcome evaluation of treated Achilles tendon ruptures. Although used predominantly to study the knee joint ${ }^{15,16}$, its reproducibility at the ankle is described for different dynamometers, positions and modes ${ }^{17-19}$. The results may be difficult to interpret and compare because test position, type of stabilization, angular velocity and type of isokinetic device are often inconsistent ${ }^{20}$. Other factors that influence the measurement include the accuracy of the dynamometer, the test protocol, the reproducibility of the measurement

Table 1. Significant influencing factors of device independent measures.

\begin{tabular}{lll}
\hline Measures & positive & negative \\
\hline ROM & peak torque plantar flexion & percutaneous therapy \\
\hline Ratio of ROM & $\begin{array}{l}\text { peak torque plantar flexion } \\
\text { peak torque dorsal extension } \\
\text { increased sports activity }\end{array}$ & percutaneous therapy \\
\hline Heel raises & $\begin{array}{l}\text { peak torque dorsal extension } \\
\text { high body height }\end{array}$ & female \\
\hline Ratio of Heel raises & high postoperative pain \\
\hline
\end{tabular}


and factors related to the subject and the person performing the test 20,21 .

In contrast, the range of motion at the ankle joint has a good to excellent correlation between test sessions $^{8}$. This together with our findings suggests that a simple test of the range of motion can reliably replace isokinetic measurements.

The ratio of dorsal extension also showed a positive correlation with the level of sport. This parameter could objectively judge the level of sport and be used to measure rehabilitation efficiency.

The calf circumference correlated positive with peak torque parameters in dorsal extension, which is known in literature ${ }^{6,22}$. However, the calf size as not just a clinical sign of gastrocnemius muscle power. This parameter is strongly influenced by gender and height and can only be used in comparison to the unaffected side and not in comparison to absolute values. However, we did not find any significant correlation with the ratio of calf circumference. Therefore the calf circumference does not give additional information for the evaluation of treated Achilles tendon ruptures.

The heel-raise test has been recommended as a measurement of calf muscle function $8,22,23$. We found that the number of heel raises was most strongly correlated with the amount of pain indicated by the patient and the level of sport. Although it is certain that calf muscle strength and endurance play a role in heel raises, it seems that this movement also integrates many other variables. It may be an indication of the general functionality of the leg. Thus pain experienced by the patient would be the single most common and most important factor reflected in this test. In athletic adults the level of sports would be equally contributing. The additional subjective assessment of sports and pain should be therefore routinely performed.

However, range of motion negatively correlated with percutaneous surgical therapy reflecting a difference in the biomechanical outcome between percutaneous therapy and open surgery. This indicates that simple and exact clinical measurements are, if well done, more than appropriate to differentiate between various therapies. Therefore, clinicians should keep in mind that valid conclusions could be made with simplest tools: hands, goniometer and wall.

\section{Conclusion}

We conclude that simple, device independent clinical measures like active range of motion and active heel raise may be more than sufficient to evaluate the outcome after Achilles tendon ruptures. The correlations we found could be used to judge the progress in recovery of healthy and athletic adults after a unilateral Achilles tendon rupture with surgical treatment. It could be that with the help of these simple measures, the long standing question of best therapy could be resolved in future trials.

\section{Key Points}

Findings: simple, device independent clinical measures and specifically the active range of motion correlate with isokinetic measurements of calf strength and may be more than sufficient to evaluate the outcome after Achilles tendon ruptures.

Implications: the correlations could be used to easily judge the progress in recovery of healthy and athletic adults after a unilateral Achilles tendon rupture with surgical treatment.

Caution: because these analyses were performed on healthy and physically active adults, the observed relationships would need to be investigated in people with pre-existing morbidities. Future trials would have to show whether these simple measurements are enough to detect differences in treatment outcomes.

\section{Competiting interests}

We affirm that we have no financial affiliation (including research funding) or involvement with any commercial organization that has a direct financial interest in any matter included in this manuscript, except as disclosed in an attachment and cited in the manuscript. Any other conflict of interest (i.e., personal associations or involvement as a director, officer, or expert witness) is also disclosed.

\section{References}

1. Maffulli N, Waterston SW, Squair J, Reaper J, Douglas A. Changing incidence of Achilles tendon rupture in Scotland: a 15-year study. Clin J Sport Med. 1999;9(3):157-160.

2. Nyyssonen $\mathrm{T}$, Luthje $\mathrm{P}, \mathrm{Kroger} \mathrm{H}$. The increasing incidence and difference in sex distribution of Achilles tendon rupture in Finland in 1987-1999. Scand J Surg. 2008;97:272-275.

3. Metz R, Verleisdonk EJ, van der Heijden GJ, et al. Acute Achilles tendon rupture: minimally invasive surgery versus nonoperative treatment with immediate full weightbearing. A randomized controlled trial. Am J Sports Med. 2008; 36(9):1688-1694.

4. Nilsson-Helander KS, Silbernagel KG, Thomee R, et al. Acute Achilles tendon rupture: a randomized, controlled study comparing surgical and nonsurgical treatments using validated outcome measures. Am J Sports Med. 2010;38(11):21862193.

5. Willits K, Amendola A, Bryant D, et al. Operative versus nonoperative treatment of acute Achilles tendon ruptures: a multicenter randomized trial using accelerated functional rehabilitation. J Bone Joint Surg Am. 2010;92(17):2767-2775.

6. Fitzpatrick R, Davey C, Buxton MJ, et al. Evaluating patientbased outcome measures for use in clinical trials. Health Technol Assess. 1998;2:i-iv, 1-74.

7. Kearney RS, Costa ML. Current concepts in the rehabilitation of an acute rupture of the tendo Achillis. J Bone Joint Surg Br. 2012;94(1):28-31.

8. Möller M, Lind K, Styf J, Karlsson J. The Reliability of isokinetic testing of the ankle joint and heel raise for endurance. Knee Surg Sports Traumatol Arthrosc. 2005; 13(1):60-71.

9. Kearney RS, Achten J, Lamb SE, Plant C, Costa ML. A Systematic Review of Patient-reported Outcome Measures Used to Assess Achilles Tendon Rupture Management: What's Be- 
ing Used and Should We Be Using It? Brit J Sport Med. 2012 Dec;46(16):1102-1109.

10. Chester R, Costa ML, Shepstone L, Donell ST. Reliability of isokinetic dynamometry in assessing plantar flexion torque following Achilles tendon rupture. Foot Ankle Int. 2003;24(12):909-915.

11. Padulo J, Oliva F, Frizziero A, Maffulli N. Muscles, Ligaments and Tendons Journal. Basic principles and recommendations in clinical and field science reasearch. MLTJ. 2013;4:250-252.

12. Webb JM, Bannister GC. Percutaneous repair of the ruptured tendo Achillis. J Bone Joint Surg. 1999;81-B:877-880.

13. Tibshirani $R$. The lasso method for variable selection in the Cox model. Stat Med. 1997;16(4):385-395.

14. Kyung M, Gill J, Ghosh M, Casella G. Penalized regression, standard errors, and Bayesian lassos. Bayesian Anal. 2010 5(2):369-411.

15. Capranica L, Battenti M, Demarie S, Figura F. Reliability of isokinetic knee extension and flexion strength testing in elderly women. J Sports Med Phys Fitness. 1998;38:169-176.

16. Li RC, Maffulli N, Hsu YC, Chan KM. Isokinetic strength of the quadriceps and hamstrings and functional ability of anterior cruciate deficient knees in recreational athletes. $\mathrm{Br} J$ Sports Med. 1996;30:161-164.
17. Andersen $\mathrm{H}$. Reliability of isokinetic measurements of ankle dorsal and plantar flexors in normal subjects and in patients with peripheral neuropathy. Arch Phys Med Rehabil. 1996;77:265-268.

18. Karnofel H, Wilkinson K, Lentell G. Reliability of isokinetic muscle testing at the ankle. J Orthop Sports Phys Ther. 1989; 11:150-154.

19. Wennerberg D. Reliability of an isokinetic dorsiflexion and plantar flexion apparatus. Am J Sports Med. 1991;19:519-522.

20. Cox PD. Isokinetic strength testing of the ankle: a review. Physiother Can. 1995;47:97-106.

21. Holmbäck AM, Porter MM, Downham D, Lexell. Reliability of isokinetic ankle dorsiflexor strength measurements in healthy young men and women. Scand J Rehabil Med. 1999;31:229239.

22. Möller M, Lind K, Movin T, Karlsson J. Calf muscle function after Achilles tendon rupture. A prospective, randomised study comparing surgical and non-surgical treatment. Scand J Med Sci Sports. 2002;12:9-16.

23. Lunsford BR, Perry J. The standing heel-rise test for ankle plantar flexion: criterion for normal. Phys Ther. 1995;75:694698. 